\title{
An Analytical Prediction Model of Balanced and Unbalanced Faults in Doubly-Fed Induction Machines
}

\author{
Frederic Maurer, Trond Leiv Toftevaag, Membership, \\ and Jonas Kristiansen Nøland, Membership
}

Post Conference Paper Ref. [22]

\begin{abstract}
This paper presents the exact transient solution to the unbalanced and balanced faults in the doubly-fed induction machine (DFIM). Stator currents, rotor currents, and stator fluxes have been validated using simulation and experiment. The work is meant to strengthen and fasten the predictability of large DFIMs in the design stage to comply with mechanical constraints or grid fault issues. Moreover, the analytical approach reduces the computational costs of large-scale stability studies and is especially suited to the initial phase where a plethora design computations must be carried out for the DFIM before it is checked for its transient interaction with the power system. The possibility to dynamically estimate DFIM performance is simplified by original equations derived from first principles. First, a case study of a large $265.50 \mathrm{MVA}$ DFIM is used to verify the proposed "large machine approximation" using simulation, achieving an exact match. Then, laboratory measurements were conducted on a $10.96 \mathrm{kVA}$ and a $1.94 \mathrm{kVA}$ DFIM to validate the transient current peaks predicted in the proposed analytic expressions for two-phase and three-phase faults, respectively.
\end{abstract}

Index Terms-Transient analytical modeling, doubly-fed induction machines (DFIMs), unbalanced short-circuits.

\section{INTRODUCTION}

$\mathbf{T}$ HE doubly-fed induction machine (DFIM) is a topology that is widely applied in a variety of power generation facilities, such as hydropower [1]-[5] and wind power [6][8] applications. In large facilities, there are concerns related to the mechanical design, the control design, as well as to the interaction with the power system [9], where transients can propagate. Constraining the armature currents and torque transients are critical machine-related issues, as their peak values influence the mechanical design and grid connection design. The DFIM must withstand these stresses without any damages, e.g., mechanical vibrations in the winding overhang of large DFIMs.

Manuscript received June 16, 2021.

Frederic Maurer, Trond Leiv Toftevaag and Jonas Kristiansen Nøland are with the Department of Electric Power Engineering, Norwegian University of Science and Technology (NTNU), Trondheim, 7034, Norway, (e-mail: frederic.maurer@ntnu.no).

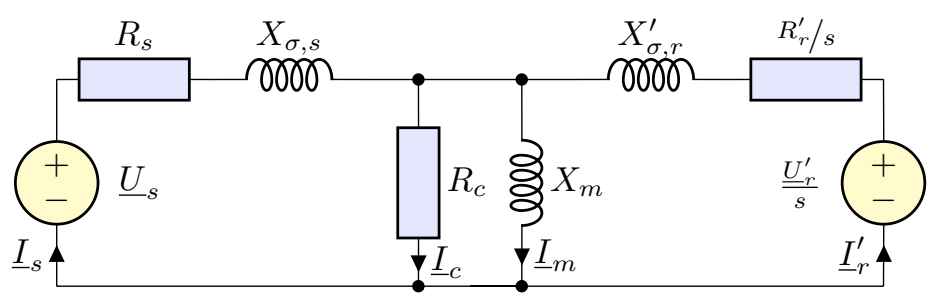

Fig. 1. Equivalent schematic diagram of the DFIM studied herein.

The rotor slip can vary from $-10 \%$ to $10 \%$ (e.g., in hydropower), or as much as from $-30 \%$ to $30 \%$ (e.g., in wind power). It is, therefore, likely that the DFIM will experience vibration, even during normal operation. For instance, rotor harmonics [10] or even supply induced harmonics [11] can lead to vibrations. Therefore, a user-friendly and simplified analytical approach is needed to give a go/no-go decision for large DFIMs in the design stage. In fact, a detailed numerical model would require huge computational efforts, e.g., finite element (FE) magnetic and mechanical calculation. The main contribution of this paper is formulating an exact analytical method to significantly reduce the computations needed to estimate peak transient torque and currents occurring in DFIMs, where FE is merely used to extract parameters prior to machine construction.

From the grid perspective, the phenomena occurring during a fault-ride through (FRT) event are much more relevant [12][16]. In these cases, the grid owner normally requires that the machine can stay connected to the grid after several transient events that might occur. However, the amplitudes of these phenomena are generally lower than for a terminal shortcircuit fault (balanced or unbalanced). They do not impact the mechanical design of the generator as the more severe, local ones. Grid faults are usually simulated in numerical simulation environments, such as SIMSEN (http://simsen.epfl.ch), as the system topology might be different for every project.

Recently, simplified models have been proposed for the DFIM under steady-state and transient operations [17], [18], without considering the short-circuit case. Reference [19] presented a harmonics analysis of the double-output induction 
generator that also overlooked the short-circuit impact. Other applications of transient models include DFIM controller improvements [20], as well as reducing the computational burden of transient stability studies [21].

This paper takes the transient domain a step further, with an earlier preliminary work already taken as a basis [22]. A complete set of dynamic equations for the induction machine was presented in [23], whereas the well-known expressions for an induction machine were already provided in [24]. However, both of these did not consider the specific case of DFIMs under terminal short-circuits. The original contribution of this study lies in the transient equations for DFIM that are applicable for the "large machine approximation" (refer to [24] for its definition), which are further derived based on the work presented by [23], and specifically adapted for a DFIM. In addition, the exact equations for predicting the unbalanced two-phase fault are also included in this paper, which is in itself a contribution. The analytic expressions also provide frequency harmonic information, which can be effectively used to ensure that a severe transient might trigger no mechanical eigenmodes over the complete operating range of a large DFIM. Compared to [22], this paper added an experimental validation of the expression developed in the conference, provided a complete derivation of the equation set, and extended the range of use to smaller power machines $(2 \mathrm{kVA}$ and $11 \mathrm{kVA})$.

As a first step, this study focuses merely on the machine side and the validation of the transient expressions for a large DFIM. Further studies should integrate grid and converter interactions as well. Analytical expressions have significant time and integration advantages compared to numerical simulation, especially in the design process of large size DFIG normally requiring between 50-100 iterations (e.g., that number can easily reach up to 1000 simulations when several different operation points must be considered). Therefore, saving time in the transient analysis is highly appreciated.

Several handpicked three-phase, star-connected DFIMs (insulation class F) are studied in this paper. One large $265 \mathrm{MVA}$ machine have been chosen for the theoretical study, which is the highest-rated DFIM in construction, while M2 and M3 are low-rated machines available in the laboratory. Tables I and II give the rated data and equivalent parameters of the machine M1 used in the theoretical analysis, made in comparison with SIMSEN. In addition, Tables II and III provide the rated data and estimated parameter values (from measurements) ${ }^{1}$ of the machines M2 and M3 used in experimental verification. Fig. 2 present the measured open-circuit characteristic of the machine M2 and M3 with an open rotor. It shows how the stator reactance $\left(X_{s}\right)$ depend on the armature voltage level, where unsaturated values are larger than those in Table III.

\footnotetext{
${ }^{1}$ The leakage reactance is obtained using the classical approach presented in the norm IEEE 112, which permits obtaining the sum of both reactances. Then according to this norm, the results can be divided by two to obtain each reactance, i.e., that is why both are equal. As the DFIM under study is the wound-rotor type, this approach is reasonable and leads to "acceptable results" as both slots should be more or less the same. Another option would have been to compute them using classical analytical computation â but as we did not find any drawing or electrical computation, this path is not practicable.
}

TABLE I

DFIM'S RATED DATA - M1, M2 \& M3

\begin{tabular}{lllll}
\hline \hline Symbol & \multirow{2}{*}{ Quantity } & M1 value & M2 value & M3 value \\
\hline$S$ & apparent power & $265.50 \mathrm{MVA}$ & $10.96 \mathrm{kVA}$ & $1.94 \mathrm{kVA}$ \\
$U_{s}$ & stator voltage & $18 \mathrm{kV}$ & $230 \mathrm{~V}$ & $400 \mathrm{~V}$ \\
$I_{s}$ & stator current & $8.5 \mathrm{kA}$ & $27.5 \mathrm{~A}$ & $2.8 \mathrm{~A}$ \\
$n$ & mech. speed & $158.51 \mathrm{rpm}$ & $1430 \mathrm{rpm}$ & $1420 \mathrm{rpm}$ \\
$f_{s}$ & stator freq. & $50 \mathrm{~Hz}$ & $50 \mathrm{~Hz}$ & $50 \mathrm{~Hz}$ \\
\hline \hline
\end{tabular}

TABLE ॥

Simulated DFim - Calculated Parameters - Machine M1

\begin{tabular}{llll}
\hline \hline Symbol & \multicolumn{1}{c}{ Quantity } & SI value & PU value \\
\hline$R_{s}$ & stator resistance & $4.5056 \mathrm{~m} \Omega$ & $0.003692 \mathrm{pu}$ \\
$R_{r}^{\prime}$ & rotor resistance & $1.9364 \mathrm{~m} \Omega$ & $0.001587 \mathrm{pu}$ \\
$X_{s}$ & stator reactance (sat.) & $2.0912 \Omega$ & $1.7136 \mathrm{pu}$ \\
$X_{m}$ & magnetizing reactance (sat.) & $1.9387 \Omega$ & $1.5887 \mathrm{pu}$ \\
$X_{\sigma, s}$ & stator leakage reactance & $0.1525 \Omega$ & $0.1250 \mathrm{pu}$ \\
$X_{\sigma, r}^{\prime}$ & rotor leakage reactance & $0.1957 \Omega$ & $0.1604 \mathrm{pu}$ \\
\hline \hline
\end{tabular}

TABLE III

Experimental DFIMS - MeAsured Parameters - M2 \& M3

\begin{tabular}{llll}
\hline \hline Symbol & \multicolumn{1}{c}{ Quantity } & M2 value & M3 value \\
\hline$R_{s}$ & cold stator resistance & $144.92 \mathrm{~m} \Omega$ & $8.40 \Omega$ \\
$R_{r}^{\prime}$ & cold rotor resistance & $231.4 \mathrm{~m} \Omega$ & $10.65 \Omega$ \\
$R_{c}$ & core loss resistance & $125.88 \Omega$ & $842.86 \Omega$ \\
$X_{s}$ & stator reactance (sat.) & $7.4800 \Omega$ & $133.39 \Omega$ \\
$X_{m}$ & magnetizing reactance (sat.) & $7.2175 \Omega$ & $117.32 \Omega$ \\
$X_{\sigma, s}$ & stator leakage reactance & $262.53 \mathrm{~m} \Omega$ & $16.07 \Omega$ \\
$X_{\sigma, r}^{\prime}$ & rotor leakage reactance & $262.53 \mathrm{~m} \Omega$ & $16.07 \Omega$ \\
\hline \hline
\end{tabular}

The remainder of the paper is organized as follows. Section II presents the modeling hypothesis and its assumptions before the transient analytical expressions of the DFIM are derived in Section III. Then, in Section IV, the torque-estimating equations are validated against a numerical simulation model. Finally, Section V validates the analytically predicted transient current peaks before Section VI concludes the paper. Appendix $A$ and $B$ provide additional details to the analytical development.

\section{Theoretical Framework AND Assumptions}

There are some important assumptions taken on board in our work, which are common for transient analysis of large AC machines [24], [25]. The resistance values are taken as constants for a given operating temperature [24]. Moreover, the saturation of the main inductance that may exist prior to the faulty condition is considered by re-adjusting the main inductance to the voltage level prior to the transient event [24]-[26]. These quantities are governed by the saturation of the stator and rotor leakage reactance, where one method postulates to artificially reduce the leakage reactances by 10 $20 \%$, depending on the initial saturation level of the machine 

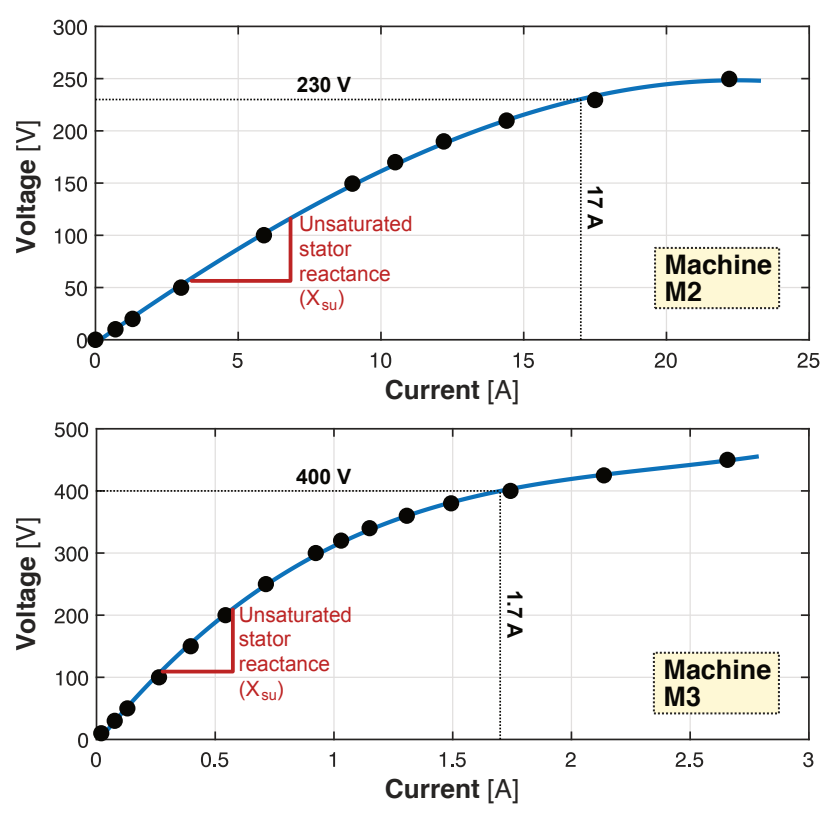

Fig. 2. Standstill open-circuit characteristics of the experimental M2 $(10.96 \mathrm{kVA})$ and M3 $(1.94 \mathrm{kVA})$ with open rotor circuit. Line-to-line stator voltage plotted against stator current.

[25], to account for this phenomenon. In general, the DFIM can be considered in steady-state with constant torque just before the short-circuit event occurs and as having constant speed during the short-circuit (i.e., ideally high inertia). This hypothesis basically holds for any large DFIM due to its rotor construction leading to an inertia constant $>5 \mathrm{~s}$ in all cases.

The voltage-sourced inverter (VSI) is modeled with a constant voltage source so that its influence is neglected during the short-circuit [24]. In order to stick with the main focus of the work, no further grid influence is considered at this stage. The VSI is assumed to continue to provide the same rotor voltage as before the transient, even though the semiconductors cannot always deliver this. However, in our downscaled experiments for M2 and M3, the assumption holds. In large-scale reality, there is a resistive crowbar going into operation when the machine and/or the VSI protection decides to fire it [26]. This firing is done to limit the current in the VSI. It takes about $5-10 \mathrm{~ms}$ to fire the crowbar, implying that the first and most important current and torque peak will happen without the impact of the crowbar. In the case of the firing of the crowbar, the rotor voltage drops down to zero and is replaced by the resistive crowbar. In order to model the described case, the rotor resistance can be adapted to reflect the crowbar resistance, and the firing of the crowbar is modeled by a rotor voltage step towards zero [26]. However, the detailed action of the crowbar is beyond the scope of this work, but all equations have been derived in a way that it will be very easy to perform that last step on top of our analysis.

The equivalent diagram of the DFIM presented in [24] is recalled in Fig. 1. It follows by Kirchoffs curren law (KCL) in complex variables that

$$
\underline{I}_{m}=\underline{I}_{s}-\underline{I}_{c}+\underline{I}_{r}^{\prime}
$$

The mathematical development is performed in the rotating dq-frame to allow exact analytical treatment, while symmetrical component only allow approximated analytical treatment. Moreover, one can easily deduce the voltage equations from Kirchoff's voltage law (KVL), yielding

$$
\begin{gathered}
\underline{U}_{s}=R_{s} \underline{I}_{s}+\mathbf{j}\left(X_{\sigma, s}+X_{h}\right) \underline{I}_{s}+X_{h} \underline{I}_{r}^{\prime} \\
\frac{\underline{U}_{r}^{\prime}}{s}=\frac{R_{r}^{\prime}}{s} \underline{I}_{r}^{\prime}+\mathbf{j}\left(X_{\sigma, r}^{\prime}+X_{h}\right) \underline{I}_{r}^{\prime}+X_{h} \underline{I}_{s}
\end{gathered}
$$

which have already been established in [24]. Eqs. (2) and (3) are formulated in transient mode, replacing their Laplaceform with time derivative, written in per-unit and omitting the underline (representing the space vector) as

$$
\begin{aligned}
& u_{s}=R_{s} i_{s}+\left(\frac{d}{d t}+\mathbf{j} \omega_{s}\right) \psi_{s}, \\
& u_{r}=R_{r} i_{r}+\left(\frac{d}{d t}+\mathbf{j} \Delta \omega\right) \psi_{r},
\end{aligned}
$$

where the '-sign is omitted not to create confusion for the transient equation $\left(L_{\mathrm{s}}^{\prime}\right.$ and $\left.L_{\mathrm{r}}^{\prime}\right)$ of the inductances. The slip $(s)$ incorporated into the definition of $\Delta \omega=s \omega_{\mathrm{s}}$, yielding

$$
\Delta \omega=\omega_{s}-\omega_{\text {mech }}=\omega_{s}-\omega_{s}(1-s)=s \omega_{s} .
$$

The fluxes in eqs. (4) and (5), with stator subscript 's' and rotor subscript ' $r$ ', are given by

$$
\begin{aligned}
& \psi_{s}=L_{s} i_{s}+L_{m} i_{r} \\
& \psi_{r}=L_{m} i_{s}+L_{r} i_{r}
\end{aligned}
$$

where $L_{s}=L_{\sigma s}+L_{m}$ and $L_{r}=L_{\sigma r}+L_{m}$.

\section{FUndamental DFIM tRANSIENT EQUATIONS}

This section takes the basics and the assumptions of Section II onboard, and formulates the transient expressions for DFIM analysis. The initial conditions are firste defined before the transient equations for the currents and flux linkages are derived. Finally, the transient torque expressions provided and the behavior prediction under unbalanced fault conditions are presented.

The derivation of the transient equations has been based on [24], which deviates from the alternative one presented in [23]. In predicting the initial state of the DFIM transients, some initial conditions (written with the subscript 'o') are needed. The stator voltage $u_{\mathrm{s}}$, the speed $n$ and the mechanical power $p_{\text {mec }}$ are known. From these three parameters, one can deduce the initial values of the stator current $\left(i_{\mathrm{s} 0}\right)$, rotor $\left(i_{\mathrm{r} 0}\right)$ current and rotor voltage $\left(u_{\mathrm{r} 0}\right)$. For the stator and rotor fluxes (used to calculate the torque) their initial conditions are given by

$$
\begin{aligned}
& u_{s o}=R_{s} i_{\mathrm{s} 0}+\mathbf{j} \omega_{s} \psi_{s o}, \\
& u_{r o}=R_{r} i_{r o}+\mathbf{j} \Delta \omega \psi_{r o},
\end{aligned}
$$

where eq. (10) is written in the stator reference frame as already stated. 


\section{A. Transient Equations for the Stator Currents}

Eqs. (4) and (5) are compactly reformatted by setting the derivator $d / d t$ equal to the operator $p$, yielding

$$
\begin{aligned}
& u_{s}=R_{s} i_{s}+\left(p+\mathbf{j} \omega_{s}\right) \psi_{s} \\
& u_{r}=R_{r} i_{r}+(p+\mathbf{j} \Delta \omega) \psi_{r} .
\end{aligned}
$$

Then, eq. (8), can be inserted into eq. (12) then solved to obtain the rotor current $\left(i_{r}\right)$ as follows

$$
i_{\mathrm{r}}=\frac{u_{\mathrm{r}}-(p+j \Delta \omega) L_{m} i_{\mathrm{s}}}{R_{r}\left(1+(p+j \Delta \omega) T_{\mathrm{r}}\right)},
$$

where $T_{r}=L_{r} / R_{r}$. Eq. (7) can be inserted into eq. (11) and combined with $i_{\mathrm{r}}$ from eq. (13), yielding

$$
\begin{aligned}
u_{\mathrm{s}}= & \frac{p^{2}+p\left(\frac{T_{\mathrm{s}}+T_{\mathrm{r}}}{T_{\mathrm{s}}^{\prime}+T_{\mathrm{r}}^{\prime}}+j\left(\omega_{\mathrm{s}}+\Delta \omega\right)\right)}{L_{\mathrm{r}}\left(1 / T_{\mathrm{r}}+(p+j \Delta \omega)\right)} i_{\mathrm{s}} \\
+ & \frac{\frac{1}{T_{\mathrm{s}} \cdot T_{\mathrm{r}}^{\prime}}\left(1+j \Delta \omega T_{\mathrm{r}}+j \omega_{\mathrm{s}} T_{\mathrm{s}}\left(1+j \Delta \omega T_{\mathrm{r}}^{\prime}\right)\right)}{L_{\mathrm{r}}\left(1 / T_{\mathrm{r}}+(p+j \Delta \omega)\right)} i_{\mathrm{s}} \\
& +\frac{L_{\mathrm{m}}}{L_{\mathrm{r}}} \frac{\left(p+j \omega_{\mathrm{s}}\right)}{1 / T_{\mathrm{r}}+(p+j \Delta \omega)} u_{\mathrm{r}},
\end{aligned}
$$

where $T_{s}=L_{s} / R_{s}, T_{\mathrm{s}}^{\prime}=L_{s}^{\prime} / R_{s}$ with $L_{s}^{\prime}=L_{\sigma, s}+$ $L_{m} L_{\sigma, r} / L_{r}$ and $T_{\mathrm{r}}^{\prime}=L_{r}^{\prime} / R_{s}$ with $L_{r}^{\prime}=L_{\sigma, r}+L_{m} L_{\sigma, s} / L_{s}$. The numerator of eq. (14) is a second order polynom and its simplification using the "large machine approximation" is detailed in Section III-D.

Eq. (14) can also be expressed for the stator current, yielding

$$
\begin{aligned}
i_{\mathrm{s}}= & \frac{L_{\mathrm{r}}}{L_{\mathrm{s}} L_{\mathrm{r}}^{\prime}} \frac{p+1 / T_{\mathrm{r}}+j \Delta \omega}{\left(p+1 / T_{\mathrm{s}}^{\prime}+j \omega_{\mathrm{s}}\right)\left(p+1 / T_{\mathrm{r}}^{\prime}+j \Delta \omega\right)} u_{\mathrm{s}} \\
& +\frac{L_{\mathrm{m}}}{L_{\mathrm{r}}} \frac{p+j \omega_{\mathrm{s}}}{\left(p+1 / T_{\mathrm{s}}^{\prime}+j \omega_{\mathrm{s}}\right)\left(p+1 / T_{\mathrm{r}}^{\prime}+j \Delta \omega\right)} u_{\mathrm{r}} .
\end{aligned}
$$

The transient equation of the stator current can be found by applying an inverse Laplace-transform to the equation. In fact, it is not straightforward to find approximate equation for $i_{\mathrm{s} 0}$, $i_{\mathrm{s} 1}$ and $i_{\mathrm{s} 2}$ (cf. Section III-E for the definition of these currents) as $\Delta \omega$ has a large amplitude variation and that the equations are not linear in terms of $\Delta \omega$ [26]. The prediction of the current calculation can be found using the exact solution to obtain the zeros of the numerator of eq. (14) as well as the coefficients $i_{\mathrm{s} 0}, i_{\mathrm{s} 1}$ and $i_{\mathrm{s} 2}$.

\section{B. Transient Equations for the Stator Flux}

The flux eqs. (7) and (8) can be expressed in matrix-form. Inverting the matrix will provide equations of the currents in terms of the fluxes. The stator current $\left(i_{s}\right)$ becomes

$$
i_{\mathrm{s}}=\underbrace{\frac{1}{L_{\mathrm{s}}^{\prime}}}_{\alpha} \psi_{\mathrm{s}}-\underbrace{\frac{L_{\mathrm{m}}}{L_{\mathrm{s}}^{\prime} L_{\mathrm{r}}}}_{-\beta} \psi_{\mathrm{r}}=\alpha \psi_{\mathrm{s}}+\beta \psi_{\mathrm{r}},
$$

and for the rotor current one obtain

$$
i_{\mathrm{r}}=-\underbrace{\frac{L_{\mathrm{m}}}{L_{\mathrm{s}}^{\prime} L_{\mathrm{r}}}}_{-\gamma} \psi_{\mathrm{s}}+\underbrace{\frac{1}{L_{\mathrm{r}}^{\prime}}}_{\delta} \psi_{\mathrm{r}}=\gamma \psi_{\mathrm{s}}+\delta \psi_{\mathrm{r}},
$$

where $L_{\mathrm{s}}^{\prime}=\left(L_{\mathrm{s}} L_{\mathrm{r}}-L_{\mathrm{m}}^{2}\right) / L_{\mathrm{r}}$, and $L_{\mathrm{r}}^{\prime}=\left(L_{\mathrm{s}} L_{\mathrm{r}}-L_{\mathrm{m}}^{2}\right) / L_{\mathrm{s}}$. Note that $\beta=\gamma$, conveniently they were defined as two separate variables. Applying eq. (17) into the rotor voltage eq. (12), and solving for the rotor flux $\left(\psi_{\mathrm{r}}\right)$ leads to

$$
\psi_{\mathrm{r}}=\frac{u_{\mathrm{r}}-R_{\mathrm{r}} \gamma \psi_{\mathrm{s}}}{R_{\mathrm{r}} \delta+(p+j \Delta \omega)} .
$$

The resulting eq. (18) can be incorporated into the stator voltage eq. (11), then solved in terms of $\psi_{\mathrm{s}}$, yielding

$$
\begin{array}{r}
\psi_{\mathrm{s}}=\frac{\left(R_{\mathrm{r}} \delta+(p+j \Delta \omega)\right) u_{\mathrm{s}}-R_{\mathrm{s}} \beta u_{\mathrm{r}}}{\left(R_{\mathrm{s}} \alpha+\left(p+j \omega_{\mathrm{s}}\right)\right)\left(R_{\mathrm{r}} \delta+(p+j \Delta \omega)\right)-R_{\mathrm{s}} R_{\mathrm{r}} \beta \gamma} \\
\psi_{\mathrm{s}}=\frac{R_{\mathrm{r}} \delta+(p+j \Delta \omega)}{\left(R_{\mathrm{s}} \alpha+\left(p+j \omega_{\mathrm{s}}\right)\right)\left(R_{\mathrm{r}} \delta+(p+j \Delta \omega)\right)-R_{\mathrm{s}} R_{\mathrm{r}} \beta \gamma} u_{\mathrm{s}} \\
-\frac{R_{\mathrm{s}} \beta}{\left(R_{\mathrm{s}} \alpha+\left(p+j \omega_{\mathrm{s}}\right)\right)\left(R_{\mathrm{r}} \delta+(p+j \Delta \omega)\right)-R_{\mathrm{s}} R_{\mathrm{r}} \beta \gamma} u_{\mathrm{r}} .
\end{array}
$$

\section{Transient Equation for the Rotor Current}

The stator voltage equation, eq. (11), is solved to obtain $i_{\mathrm{s}}$, which will be inserted into the rotor current equation, eq. (13), which will be solved to obtain the desired transfer function. The result of these manipulations can then be inserted into the rotor voltage equation, yielding

$$
\begin{aligned}
u_{\mathrm{r}}= & R_{\mathrm{r}}\left(1+(p+j \Delta \omega) T_{\mathrm{r}}\right) i_{\mathrm{r}}+(p+j \Delta \omega) L_{\mathrm{m}} i_{\mathrm{s}}, \\
u_{\mathrm{r}} & =\frac{R_{\mathrm{r}} R_{\mathrm{s}}\left(1+(p+j \Delta \omega) T_{\mathrm{r}}\right)\left(1+\left(p+j \omega_{\mathrm{s}}\right) T_{\mathrm{s}}\right)}{R_{\mathrm{s}}\left(1+\left(p+j \omega_{\mathrm{s}}\right) T_{\mathrm{s}}\right)} i_{\mathrm{r}} \\
& -\frac{(p+j \Delta \omega)\left(p+j \omega_{\mathrm{s}}\right) L_{\mathrm{m}}^{2}}{R_{\mathrm{s}}\left(1+\left(p+j \omega_{\mathrm{s}}\right) T_{\mathrm{s}}\right)} i_{\mathrm{r}} \\
& +\frac{(p+j \Delta \omega) L_{\mathrm{m}}}{R_{\mathrm{s}}\left(1+\left(p+j \omega_{\mathrm{s}}\right) T_{\mathrm{s}}\right)} u_{\mathrm{s}} .
\end{aligned}
$$

Eq. (22) can be formulated in terms of $i_{\mathrm{r}}$, leading to the rotor current expression. Reference [26] presents a validation using the SIMSEN numerical environment for simulating the presented rotor current equations and got a good agreement between analytical and numerical expressions.

\section{Simplification of the Poles of the Transfer Function}

Recalling the numerator of eq. (14) leads to

$$
\begin{array}{r}
p^{2}+p\left[\frac{T_{\mathrm{s}}+T_{\mathrm{r}}}{T_{\mathrm{s}}^{\prime}+T_{\mathrm{r}}^{\prime}}+j\left(\omega_{\mathrm{s}}+\Delta \omega\right)\right] \\
+\frac{1}{T_{\mathrm{s}} \cdot T_{\mathrm{r}}^{\prime}}\left[1+j \Delta \omega T_{\mathrm{r}}+j \omega_{\mathrm{s}} T_{\mathrm{s}}\left(1+j \Delta \omega T_{\mathrm{r}}^{\prime}\right)\right]=0 .
\end{array}
$$

In the field of large electrical machines, the following inequalities can be considered as fulfilled (refer to [24])

$$
T_{\mathrm{r}}>T_{\mathrm{s}}>>T_{\mathrm{r}}^{\prime}>>T_{\mathrm{s}}^{\prime}>>1 / \omega_{\mathrm{s}} .
$$



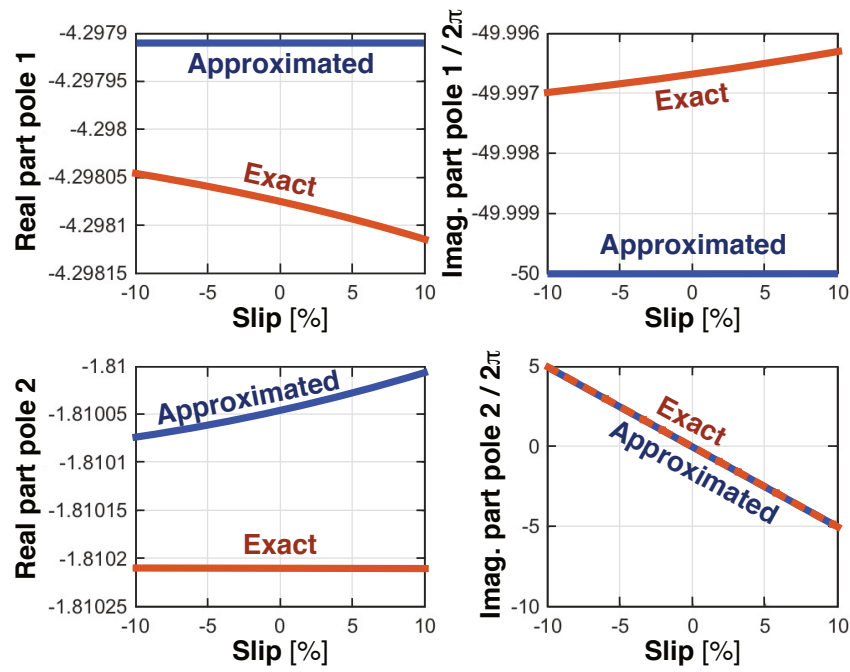

Fig. 3. Evolution of the poles $p_{1}$ and $p_{2}$ in function of $\Delta \omega$. Adapted from preliminary work [22].

The inequalities are also called the "large machine approximation". On purpose, $\Delta \omega$ was deliberately not used in the inequalities as it can change in the range $-0.1 \omega_{\mathrm{s}}$ to $0.1 \omega_{\mathrm{s}}$. It simplifies the equation of the poles and shows that the poles $\left(p_{1}\right.$ and $\left.p_{2}\right)$ can be expressed as

$$
\begin{aligned}
& p_{1}=-1 / T_{\mathrm{s}}^{\prime}-j \omega_{\mathrm{s}}, \\
& p_{2}=-1 / T_{\mathrm{r}}^{\prime}-j \Delta \omega,
\end{aligned}
$$

at leading order. Further treatment of this part can be found in [22], [26]. Fig. 3 shows the evolution if the real part and imaginary part of $p_{1}$ and $p_{2}$ for different values of $\Delta \omega$. One can see that at leading order, the poles behave like the simplified equations predicts.

\section{E. Torque Harmonics for a Three-Phase Short-Circuit, or Three-Phase Faulty Synchronization}

In order to exclude any excitation of a mechanical eigenmode in the stator or rotor of the generator, one needs to determine the frequency of the torque harmonics. From eq. (15) one can deduce that the stator current is given by

$$
\begin{aligned}
i_{\mathrm{s}} & =i_{\mathrm{s} 0}+i_{\mathrm{s} 1} \exp \left(-t / T_{\mathrm{s}}^{\prime}\right) \sin \left(\omega_{\mathrm{s}} t\right) \\
& +i_{\mathrm{s} 2} \exp \left(-t / T_{\mathrm{r}}^{\prime}\right) \sin (\Delta \omega t)
\end{aligned}
$$

where $i_{\mathrm{s} 0}, i_{\mathrm{s} 1}$ and $i_{\mathrm{s} 2}$ (the detailed expression are given in Sec. VII-A) are obtained from the corresponding transfer function. Similarly, the stator flux is obtained from the stator current, starting from eq. (20), yielding

$$
\begin{aligned}
\psi_{\mathrm{s}} & =\psi_{\mathrm{s} 0}+\psi_{\mathrm{s} 1} \exp \left(-t / T_{\mathrm{s}}^{\prime}\right) \sin \left(\omega_{\mathrm{s}} t\right) \\
& +\psi_{\mathrm{s} 2} \exp \left(-t / T_{\mathrm{r}}^{\prime}\right) \sin (\Delta \omega t),
\end{aligned}
$$

where $\psi_{\mathrm{s} 0}, \psi_{\mathrm{s} 1}, \psi_{\mathrm{s} 2}$ (the detailed expression are given in Sec. VII-B) are obtained from the corresponding transfer function. Finally, the torque $\left(T_{e m}\right)$ is given by

$$
T_{\text {em }}=\frac{3}{2} \cdot p \cdot \operatorname{Im}\left\{\psi_{\mathrm{s}}^{*} \cdot i_{\mathrm{s}}\right\} .
$$

The interesting part of the torque harmonics is to identify if there could be any resonance between the mechanical system and the foundation, i.e., it is not mandatory to calculate each component. Its frequency dependence leads to

$$
\begin{aligned}
& f_{1}=2 \Delta \omega \\
& f_{2}=\omega_{\mathrm{s}} \pm \Delta \omega \\
& f_{3}=\omega_{\mathrm{s}} \\
& f_{4}=2 \omega_{\mathrm{s}} .
\end{aligned}
$$

\section{F. Modelling the Two-Phase Short-Circuit}

In the current research literature, no exact analytical expression for the current or torque of a two-phase short-circuit in the dq-rotating frame have been proposed [27], [28]. Based on a previous works on the DC-Decay tests (i.e., two-phase short-circuit) in a synchronous machine [28], the two-phase short-circuit was characterised by the following equation

$$
u_{a}=u_{b} .
$$

Eq. (34) imply that two voltages will be clamped to each other after the short-circuit. In addition to this, if the machine is supposed to be star-connected, yields as the following relations.

$$
\begin{aligned}
u_{\mathrm{a}}+u_{\mathrm{b}}+u_{\mathrm{c}} & =0 \\
2 u_{\mathrm{a}}+u_{\mathrm{c}} & =0 .
\end{aligned}
$$

In order to obtain the voltages after the short-circuit, the reaction of the voltage on the open phase $c$ must be known or assumed known after the event. Prior to the short-circuit, the phase voltage of $c$ is given neither by the machine (through the induced voltage) or by the grid. Just after the fault (a few milliseconds after), the currents in the machine will not have any discontinuity. The currents and the flux linkages are state variables, so that the voltage in phase $c$ will remain the same. The terminal voltage of phase $c$, is not affected by the shortcircuit, will not change. As a result,

$$
u_{\mathrm{c}}=u_{\mathrm{s}} \text {, }
$$

where $u_{\mathrm{s}}$ is the stator voltage phasor before the short-circuit. In this case, it is important to keep Park's reference frame aligned with the stator voltage in order to get a real number. From this understanding, the stator voltage in the $a b c$-frame is known before and after the short-circuit, yielding

$$
\begin{array}{r}
u_{\mathrm{a}}=-u_{\mathrm{c}} / 2, \\
u_{\mathrm{b}}=-u_{\mathrm{c}} / 2, \\
u_{\mathrm{c}}=u_{\mathrm{c}} .
\end{array}
$$

After some trivial trigonometric operations, one obtains the voltage in the rotating frame, yielding

$$
\begin{aligned}
& u_{d}=-\frac{u}{2} \sin \left(2 \omega_{\mathrm{s}} t\right) \\
& u_{q}=-\frac{u}{2}\left(1+\cos \left(2 \omega_{\mathrm{s}} t\right)\right),
\end{aligned}
$$

which is an original contribution of this work and have also been validated with measurement performed on a large $\mathrm{AC}$ machine, as preliminary presented in [26]. In summary, the 
torque harmonics appearing during a two-phase short-circuit or faulty synchronisation, the technique described in this subsection uses the voltage eqs. (40) and (41).

\section{Verification of the TORQUe EQUations}

This section presents the validation of the analytical equations developed in Section III for an ASM and DFIM at rated load operation. The verification of the torque is done
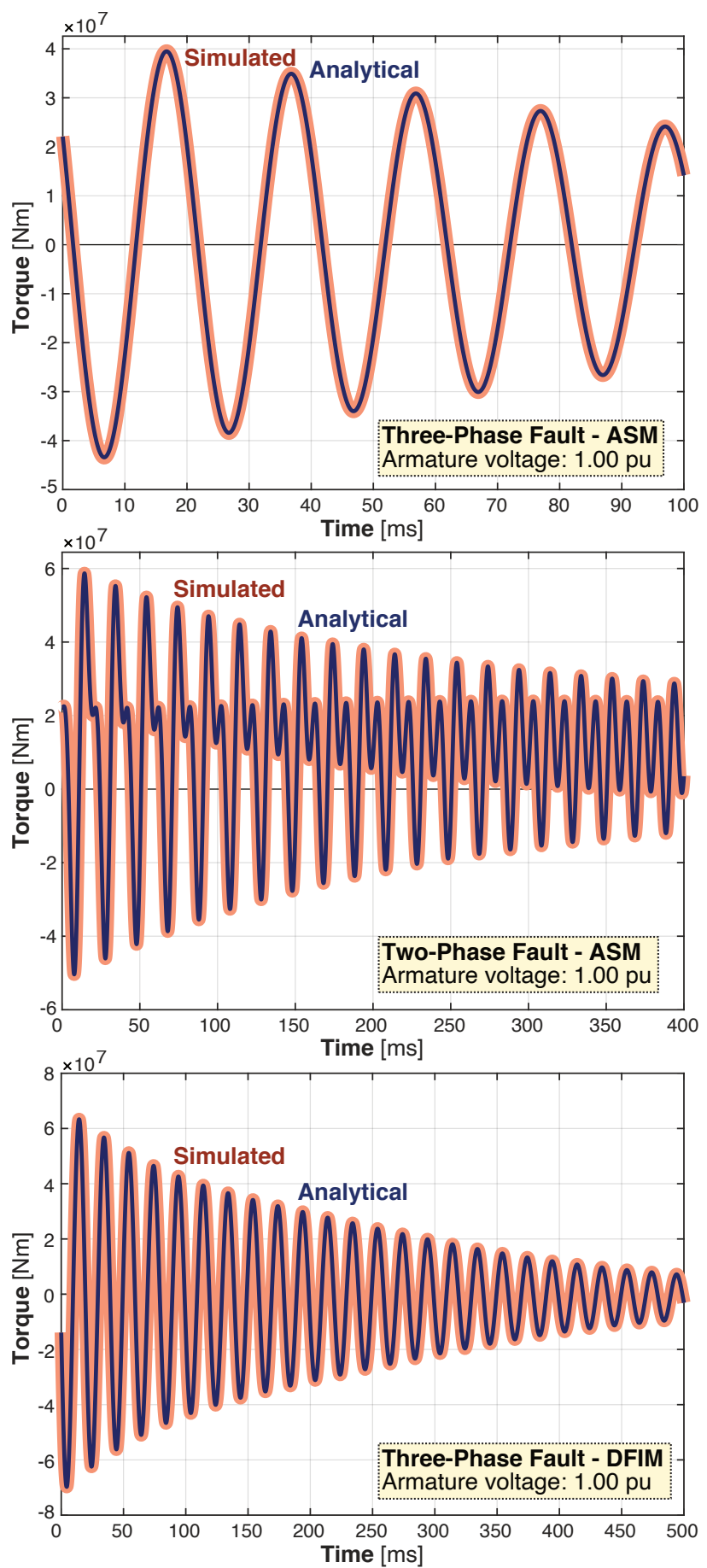

Fig. 4. Transient torque asynchronous with fault event initiated at $0 \mathrm{~ms}$, with profile given by eq. (29). ASM has short-circuited rotor, while DFIM has constant voltage-fed rotor. Steady constant torque was assumed before the short-circuit. Adapted from preliminary work [22]

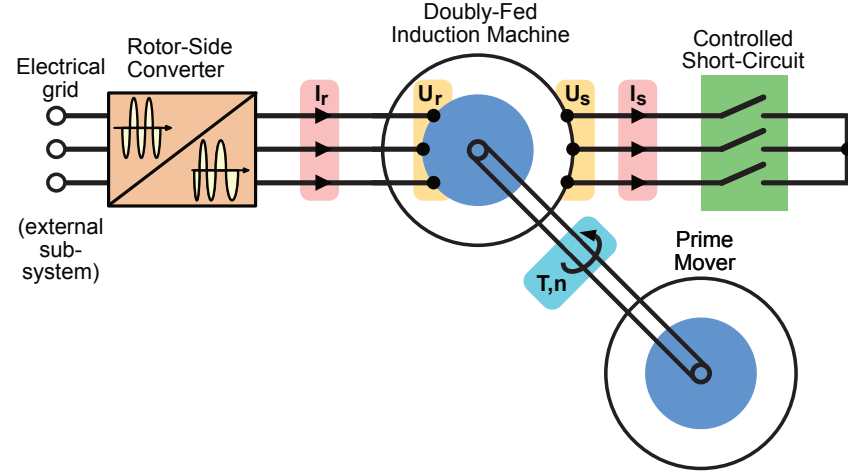

Fig. 5. Schematic description of the experimental test setup (M2 \& M3).

in the SIMSEN numerical software environment using the parameters defined in Table II (i.e., handpicked simulation case study). SIMSEN assumes an equivalent diagram as given in Fig. 1 and applies the same hypothesis as the one defined in Section II. The electrical machine is connected to the grid on both stator- and rotor-side converters and operated at rated operatino before the short-circuit. The analytical equations are compared to numerical solutions. Practically speaking, one enters the same parameters (refer to Table II) and initial conditions and compares the output. The limitations of this confirmation merely depend on the modelling hypothesis that have been proposed. Equivalent parameters for the ASM (i.e., asynchronous machine) and the DFIM (i.e., doubly-fed induction machine) were the same. The ASM is, therefore, a virtual machine, while the DFIM can be considered as the real case. The ASM case was only used to simplify the model and to have a richer validation study. The DFIM is operating at rated conditions (over-excited) before the fault, while the ASM is operating at the same equivalent operating point (same active power) before the short-circuit for both the two-phase and three-phase short-circuit. As the verification for the twophase short-circuit only depends on the stator voltage equation, it has been decided to perform it only on the ASM, i.e., the simplest case. Given this premise, the two-phase validation for the DFIM case is implicit. Fig. 4 present the transient air-gap torque curves for the ASM machine in three-phase and two-phase short-circuit, and DFIG in the case of a threephase short-circuit. Finally, it can be observed that all transient curves agrees well with the simulated curves in SIMSEN.

\section{Experimental Validation of Current Peaks}

This section extends the verification made in Section IV by providing an experimental validation. The test rig is illustrated and depicted in Figs. 5 and 6. Herein, the rotor-side converter (RSC) has a maximum transient rating of $250 \mathrm{kVA}$ and $400 \mathrm{~A}$. By intention, the stator voltage have been reduced to avoid entering into the current limitation mode of the RSC so that no coupling of the stator with the grid can be considered. In addition, the main goal of the validation is to measure shortcircuits (two-phase and three-phase) from no-load, considering the fact that a load short-circuit has the same expressions [26]. This reduction voltage also limits the transient torques, which is preferred due to the mechanical limitation in the laboratory. 


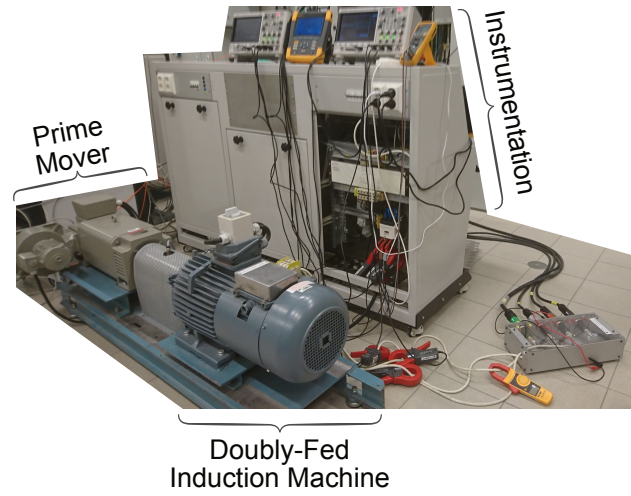

Fig. 6. Picture of experimental test rig (M2).

There was no additional rotating inertia on the shaft-line, and the rotational speed was only partially maintained by the speed regulator of the prime mover (i.e., the DC-machine). Therefore, some slight speed changes will unavoidably occur and perturbed the measurements.

Prior to the short-circuit, the machine was operated in noload condition, with the rotor fed by the RSC, with a low stator voltage. The fault is established by closing the short-circuit contactor on the stator side, which induces no redundancy (i.e., confirmed by the contactor closing curves). Then, the stator currents was recorded using a digital storage oscilloscope and plotted against the simulated transient curves. The process was similar for M2 and M3.

\section{A. Experimental Results of Machine M2}

Fig. 7 presents the three individual tests done on machine M2. Test 1 was done at $50 \%$ of rated voltage. There is small speed change leading to the observed frequency sweep (due to lack of rotating inertia installed). The frequency sweep is not present for test 2 done at $25 \%$ armature voltage. The observed current peaks confirm the computation model. For the two-phase fault, the agreement was worst for the first current peak, while a small frequency sweep due to the same above-mentioned phenomenon. However, the steadystate short-circuit current and the first peak are really well estimated, while only the second and third peak deviates from the estimated one (i.e., over-estimated). This phenomena is an issue of further work to determine if this effect is coming of the test platform, considering the simulated M1 agreed well for the whole transient envelope.

Fig. 8 shows the current decomposition for M3 (three-phase short-circuit). After the transient, which is governed by the component 1 and 2, the machine goes into sustained shortcircuit operation given by the 0 -component. It is interesting to note that the peak is driven by the difference between the components 1 and 2 .

\section{B. Experimental Results Machine M3}

Fig. 9 presents the 4 tests done on machine M3. Test 1 was done at $100 \%$ of rated voltage. For this reason, there is a significant transient speed change occurring, leading to the
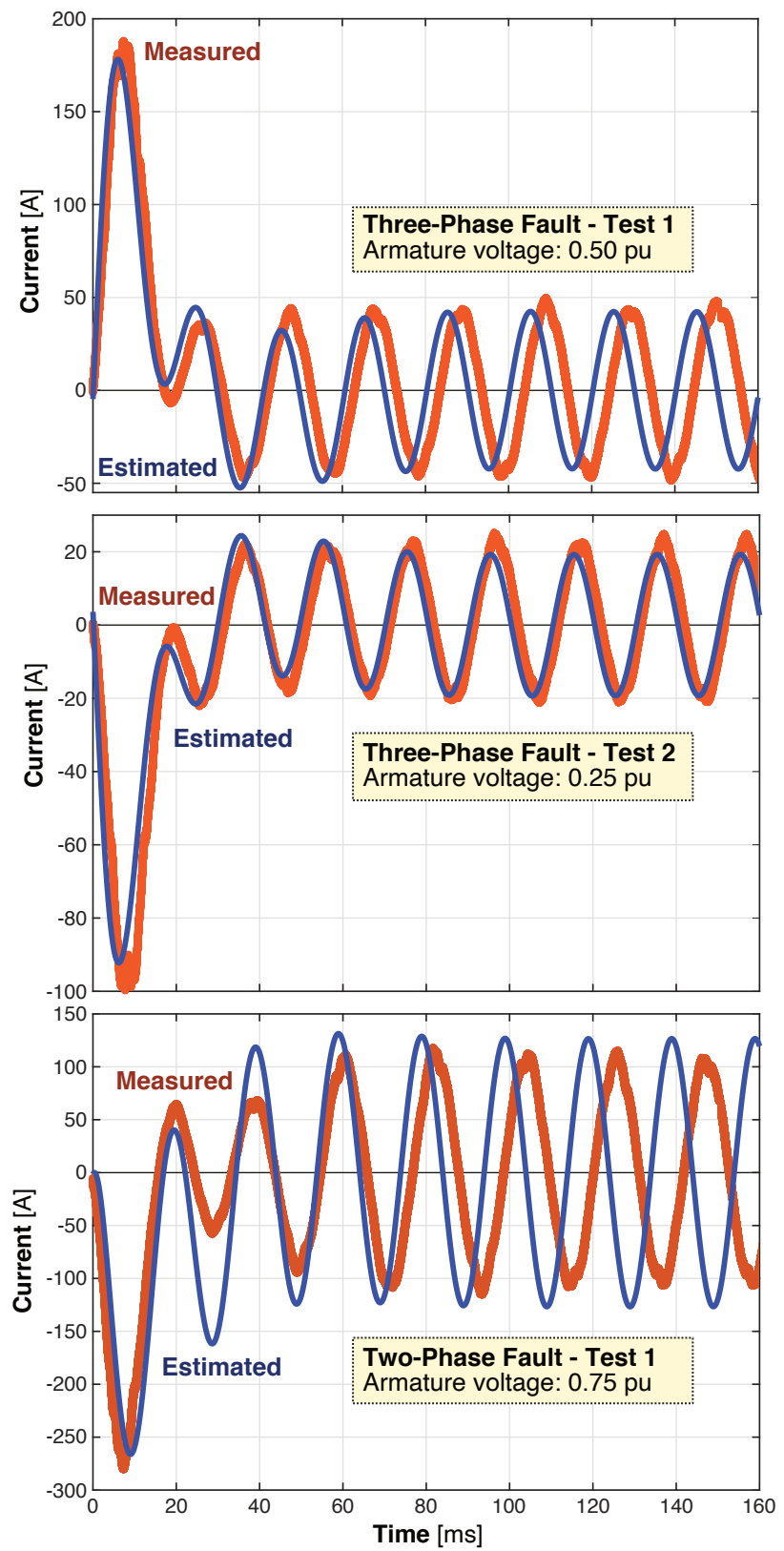

Fig. 7. Experimental results of M2 (10.96 kVA DFIM) with fault initiated at $0 \mathrm{~ms}$. Estimated curves were solved by eq. (15). Speed was $1350 \mathrm{rpm}$ ( $50 \mathrm{~Hz}$ electrical). The base armature voltage is $230 \mathrm{~V}$.

observed frequency sweep (due to lack of rotating inertia). However, the frequency sweep is not present for test 2 done at $50 \%$ armature voltage and for test 3 done at $25 \%$ of the nominal voltage. Observed for the two-phase fault, a very good agreement is seen for the first current peak and a small frequency sweep due to the same above-mentioned phenomenon. The steady-state short-circuit current is also well estimated, while only the second and third peak deviates slightly from our estimations. In general, the measurements tend to showcase that the speed change could be the main origin of errors, but other factors could also be involved. The two-phase fault has the highest peaks for same voltage.

Fig. 10 shows the current decomposition for M3 (two-phase short-circuit). After the transient, which is governed by the 


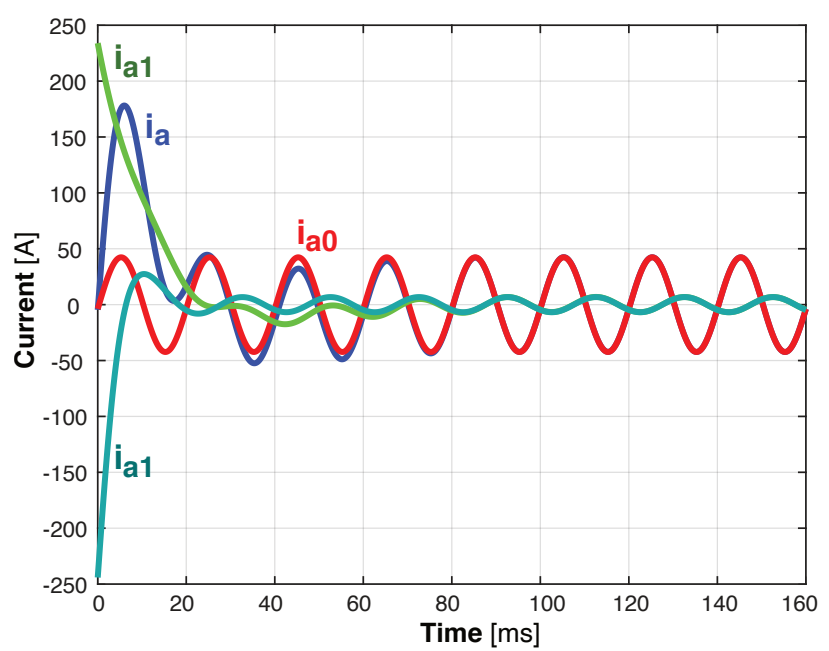

Fig. 8. Detailed computation of each stator current component of the stator three-phase short-circuit current with an armature voltage of $0.5 \mathrm{pu}$ for M2 (10.96 kVA DFIM) with fault initiated at $0 \mathrm{~ms}$. Speed was $1350 \mathrm{rpm}(50 \mathrm{~Hz}$ electrical). The base armature voltage is $230 \mathrm{~V}$.

component 1 and 2, the machine goes into sustained shortcircuit operation given by the 0-component and by the 3 component (due to the two-phase pole). It is interesting to note that the peak is driven by the difference between the components 1 and 2 .

\section{CONCLUSION}

This paper simplifies the transient faulty analysis of doublefed induction machines (DFIMs) by proposing an exact analytical model. The framework provides an accurate indication of the harmonic components in the transient and an estimation of the peak torque and currents during faulty events. The approach is in excellent agreement with a detailed numerical model. In addition, the "large machine approximation" is introduced for the analytical model, and it is shown to be valid for the case study of machine M1. Finally, two other case studies of experimentally tested machines show that the transient peaks in the armature current amplitudes correspond well with our analytical model, including balanced three-phase faults and unbalanced two-phase short-circuits, respectively. In general, the predictability of our analytic approach can be used to guide the mechanical design of large power conversion facilities, such as variable-speed hydropower and wind power plants. In future research items, complete modeling of the rotor-side converter (RSC) and its control actions can be incorporated in more detail, if needed, for the particular application.

Further work will focus on the integration with the grid, the rotor-side converter (RSC), and especially on the rotor current saturation due to the active current limitation of the RSC.

\section{ACKNOWLEDGMENT}

The authors would like to thank staff engineer EE Svein Erling Norum, Department of Electric Power Engineering, NTNU, for his valuable contributions to the laboratory experiments conducted as part of the work presented. His technical
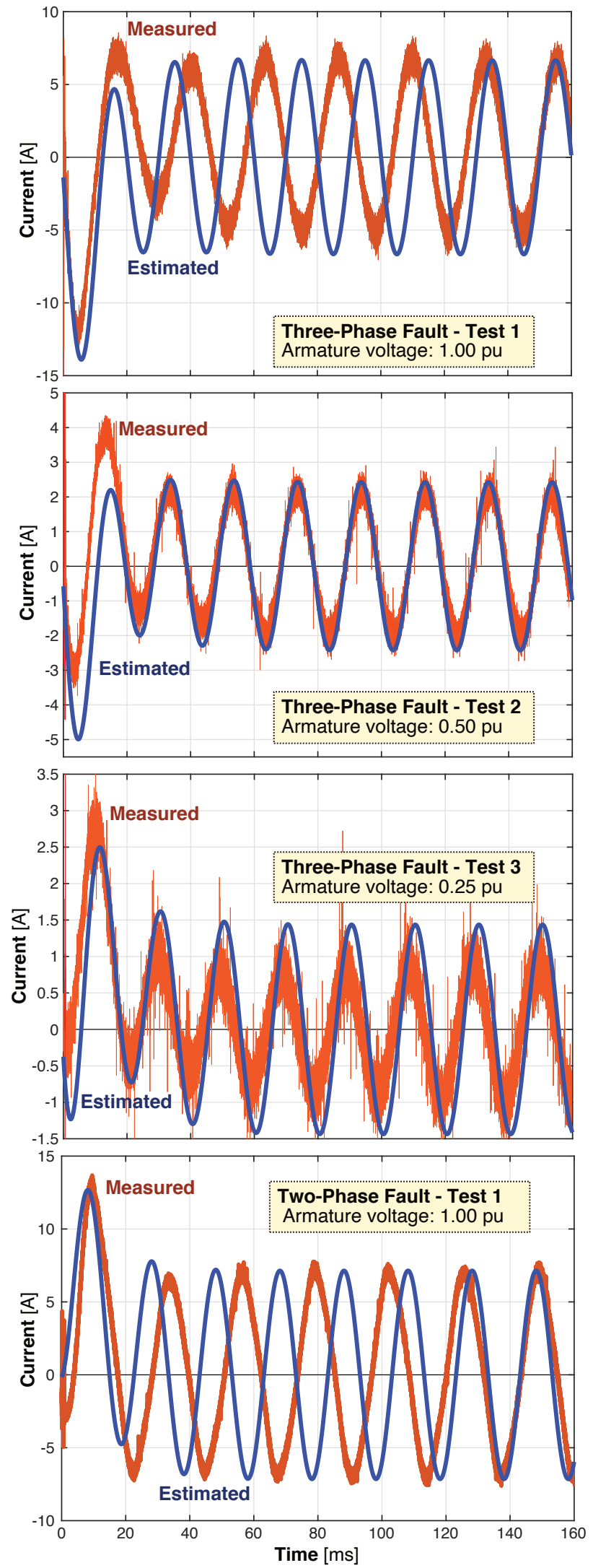

Fig. 9. Experimental results of M3 (1.94kVA DFIM) with fault initiated at $0 \mathrm{~ms}$. Estimated curves were solved by eq. (15). Speed was $1350 \mathrm{rpm}$ ( $50 \mathrm{~Hz}$ electrical). The base armature voltage is $400 \mathrm{~V}$. 


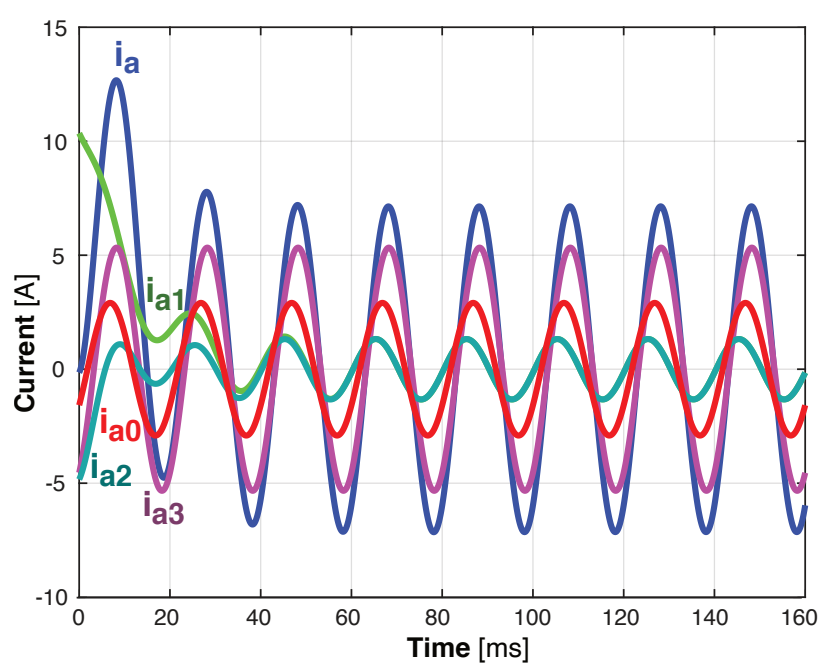

Fig. 10. Detailed computation of each stator current component of the stator three-phase short-circuit current with an armature voltage of $0.5 \mathrm{pu}$ for M3 (1.94 kVA DFIM) with fault initiated at $0 \mathrm{~ms}$. Speed was $1350 \mathrm{rpm}(50 \mathrm{~Hz}$ electrical). The base armature voltage is $400 \mathrm{~V}$.

skills in instrumentation, creativity in finding practical solutions, flexibility, and interpersonal skills have been of great importance in this context and are highly appreciated.

\section{APPENDIX}

\section{A. Expressions for the Stator Current $\left(i_{\mathrm{s} 0}, i_{\mathrm{s} 1}\right.$ and $\left.i_{\mathrm{s} 2}\right)$}

A demonstrated in [26], there are no simplified expressions for the stator current as the slip varies between $-10 \%$ and $+10 \%$. One must therefore compute the exact expression for each speed. To keep the expression readable and simple, the derivation uses matrix-expression. The equations for to obtain the 3 phases short-circuit are prenseted in detail hereafter. Due to space limitations, the derivation for the 2 phase short-circuit is left over but it follows exactly the same principle. The stator current is given for the 3phase short-circuit by

$$
\begin{aligned}
i_{\mathrm{s}} & =i_{\mathrm{s} 0}+i_{\mathrm{s} 1} \exp \left(-t / T_{\mathrm{s}}^{\prime}\right) \sin \left(\omega_{\mathrm{s}} t\right) \\
& +i_{\mathrm{s} 2} \exp \left(-t / T_{\mathrm{r}}^{\prime}\right) \sin (\Delta \omega t)
\end{aligned}
$$

where $i_{\mathrm{s} 0}, i_{\mathrm{s} 1}$ and $i_{\mathrm{s} 2}$ will be determined hereafter. Defining the zero $\left(z_{i}\right)$ of the transfer function (Eq. 15) by

$$
z_{i}=-1 / T_{\mathrm{r}}-j \Delta \omega
$$

leads to the following expression for $i_{\mathrm{s} 0}$

$$
i_{\mathrm{s} 0}=-u_{\mathrm{s} 0} \frac{z_{i}}{p_{1} * p_{2}} \frac{T_{\mathrm{r}}}{R_{\mathrm{s}} T_{\mathrm{s}} T_{\mathrm{r}}^{\prime}}
$$

where $p_{1}$ and $p_{2}$ are the pole of the transfer function (Eq. 15). $i_{\mathrm{s} 1}$ and $i_{\mathrm{s} 2}$ are given by

$$
\begin{aligned}
& i_{\mathrm{s} 1}=u_{\mathrm{s} 0} \zeta \frac{T_{\mathrm{r}}}{R_{\mathrm{s}} T_{\mathrm{s}} T_{\mathrm{r}}^{\prime}} \\
& i_{\mathrm{s} 2}=u_{\mathrm{s} 0} \eta \frac{T_{\mathrm{r}}}{R_{\mathrm{s}} T_{\mathrm{s}} T_{\mathrm{r}}^{\prime}}
\end{aligned}
$$

where $\zeta$ and $\eta$ can be obtained using a matrix-approach. The matrix approach comes from the separation of variables to be done (leading to 2 equations with 2 unkown) when inverting
Eq. 15 to transform it back to the temporal space. The matrix approach leads to $\mathrm{a} A \mathrm{x}=\mathrm{b}$ equation to be solved. Defining the b-vector as

$$
b=\left(\frac{\frac{z_{i}}{p_{1} z_{i}}}{\frac{z_{i}}{\left(p_{1} * p_{2}\right)\left(p_{1}+p_{2}\right)-1}}\right)
$$

and the A-matrix as

$$
A=\left(\begin{array}{cc}
1 & 1 \\
p_{2} & p_{1}
\end{array}\right)
$$

leads to the following expression for $\zeta$ and $\eta$

$$
\left(\begin{array}{l}
\zeta \\
\eta
\end{array}\right)=A^{-1} b
$$

\section{B. Expressions for the Stator Flux $\left(\psi_{\mathrm{s} 0}, \psi_{\mathrm{s} 1}, \psi_{\mathrm{s} 2}\right)$}

To obtain the detailed expression for the stator flux, one uses the same principle as for the stator current (refer to section VII-B for the detailed process). The stator flux is given by

$$
\begin{aligned}
\psi_{\mathrm{s}} & =\psi_{\mathrm{s} 0}+\psi_{\mathrm{s} 1} \exp \left(-t / T_{\mathrm{s}}^{\prime}\right) \sin \left(\omega_{\mathrm{s}} t\right) \\
& +\psi_{\mathrm{s} 2} \exp \left(-t / T_{\mathrm{r}}^{\prime}\right) \sin (\Delta \omega t),
\end{aligned}
$$

where $\psi_{\mathrm{s} 0}, \psi_{\mathrm{s} 1}, \psi_{\mathrm{s} 2}$ will be computed hereafter.

Defining the zero $\left(z_{\psi}\right)$ of the transfer function (Eq. 20) by

$$
z_{\psi}=-R_{r} \delta-j \Delta \omega
$$

leads to the following expression for $\psi_{\mathrm{s} 0}$

$$
\psi_{\mathrm{s} 0}=-u_{\mathrm{s} 0} \frac{z_{\psi}}{p_{1} * p_{2}}
$$

where $p_{1}$ and $p_{2}$ are the pole of the transfer function (Eq. 20). $\psi_{\mathrm{s} 1}$ and $\psi_{\mathrm{s} 2}$ are given by

$$
\begin{aligned}
& \psi_{\mathrm{s} 1}=u_{\mathrm{s} 0} \zeta_{\psi} \\
& \psi_{\mathrm{s} 2}=u_{\mathrm{s} 0} \eta_{\psi}
\end{aligned}
$$

where $\zeta_{\psi}$ and $\eta_{\psi}$ can be obtained using a matrix-approach. This approach leads to $\mathrm{a} A \mathrm{x}=\mathrm{b}$ equation to be solved. Defining the b-vector as

$$
b=\left(\begin{array}{c}
\frac{z_{\psi}}{p_{1} * p_{2}} \\
\frac{z_{\psi}}{\left(p_{1} * p_{2}\right)\left(p_{1}+p_{2}\right)-1}
\end{array}\right)
$$

and the A-matrix as

$$
A=\left(\begin{array}{cc}
1 & 1 \\
p_{2} & p_{1}
\end{array}\right)
$$

leads to the following expression for $\zeta$ and $\eta$

$$
\left(\begin{array}{c}
\zeta_{\psi} \\
\eta_{\psi}
\end{array}\right)=A^{-1} b
$$




\section{Expressions for the stator rotor $\left(i_{\mathrm{r} 0}, i_{\mathrm{r} 1}\right.$ and $\left.i_{\mathrm{r} 2}\right)$}

The rotor current is given for the 3 phase short-circuit by

$$
\begin{aligned}
i_{\mathrm{r}} & =i_{\mathrm{r} 0}+i_{\mathrm{r} 1} \exp \left(-t / T_{\mathrm{s}}^{\prime}\right) \sin \left(\omega_{\mathrm{s}} t\right) \\
& +i_{\mathrm{r} 2} \exp \left(-t / T_{\mathrm{r}}^{\prime}\right) \sin (\Delta \omega t)
\end{aligned}
$$

where $i_{\mathrm{r} 0}, i_{\mathrm{r} 1}$ and $i_{\mathrm{r} 2}$ will be determined hereafter.

Defining the zero $\left(z_{r}\right)$ of the transfer function (Eq. 22) by

$$
z_{r}=-j \Delta \omega
$$

leads to the following expression for $i_{\mathrm{r} 0}$

$$
i_{\mathrm{r} 0}=-u_{\mathrm{s} 0} \frac{z_{r}}{p_{1} * p_{2}} \frac{x_{h}}{x_{h}^{2}-x_{s} x_{r}}
$$

where $p_{1}$ and $p_{2}$ are the pole of the transfer function (Eq. 22). $i_{\mathrm{r} 1}$ and $i_{\mathrm{r} 2}$ are given by

$$
\begin{aligned}
& i_{\mathrm{r} 1}=u_{\mathrm{s} 0} \zeta_{r} \\
& i_{\mathrm{r} 2}=u_{\mathrm{s} 0} \eta_{r}
\end{aligned}
$$

where $\zeta_{r}$ and $\eta_{r}$ can be obtained using a matrix-approach. This approach leads to a $A x=b$ equation to be solved. Defining the b-vector as

$$
b=\left(\begin{array}{c}
\frac{z_{r}}{p_{1} * p_{2}} \\
\frac{z_{r}}{\left(p_{1} * p_{2}\right)\left(p_{1}+p_{2}\right)-1}
\end{array}\right)
$$

and the A-matrix as

$$
A=\left(\begin{array}{cc}
1 & 1 \\
p_{2} & p_{1}
\end{array}\right)
$$

leads to the following expression for $\zeta$ and $\eta$

$$
\left(\begin{array}{c}
\zeta_{r} \\
\eta_{r}
\end{array}\right)=A^{-1} b
$$

\section{REFERENCES}

[1] A. Joseph, K. Desingu, R. R. Semwal, T. R. Chelliah, and D. Khare, "Dynamic performance of pumping mode of $250 \mathrm{mw}$ variable speed hydro-generating unit subjected to power and control circuit faults," IEEE Trans on Energy Convers., vol. 33, no. 1, pp. 430-441, 2018.

[2] R. Kumari, K. K. Prabhakaran, K. Desingu, T. R. Chelliah, and S. V. A. Sarma, "Improved hydroturbine control and future prospects of variable speed hydropower plant," IEEE Trans. Ind. Appl., vol. 57, no. 1, pp. 941-952, 2021.

[3] K. Desingu, R. Selvaraj, A. K. Birudula, and T. R. Chelliah, "Thermal performance improvement in multi-megawatt power converters serving to asynchronous hydro generators operating around synchronous speed," IEEE Trans. Energy Convers., pp. 1-1, 2020.

[4] A. Joseph, R. Selvaraj, T. R. Chelliah, and S. V. A. Sarma, "Starting and braking of a large variable speed hydrogenerating unit subjected to converter and sensor faults," IEEE Trans. Ind. Appl., vol. 54, no. 4, pp. 3372-3382, 2018.

[5] A. Joseph, T. R. Chelliah, R. Selvaraj, and K.-B. Lee, "Fault diagnosis and fault-tolerant control of megawatt power electronic converter-fed large-rated asynchronous hydrogenerator," IEEE J. Emerg. Sel. Top. Power Electron., vol. 7, no. 4, pp. 2403-2416, 2019.

[6] M. Abdeen, H. Li, S. Kamel, A. Khaled, M. El-Dabah, M. Kharrich, and H. F. Sindi, "A recent analytical approach for analysis of subsynchronous resonance in doubly-fed induction generator-based wind farm," IEEE Access, vol. 9, pp. 68 888-68 897, 2021.
[7] X. Wang, D. Liu, and H. Polinder, "Improving annual energy production of doubly-fed induction generators," IEEE Trans. Energy Convers., pp. 1-1, 2021.

[8] P. Chao, W. Li, X. Liang, S. Xu, and Y. Shuai, "An analytical twomachine equivalent method of dfig-based wind power plants considering complete frt processes," IEEE Trans. Power Syst., pp. 1-1, 2021.

[9] M. Debouza and A. Al-Durra, "Grid ancillary services from doubly fed induction generator-based wind energy conversion system: A review," IEEE Access, vol. 7, pp. 7067-7081, 2019.

[10] Yong Liao, Li Ran, G. A. Putrus, and K. S. Smith, "Evaluation of the effects of rotor harmonics in a dfig with harmonic induced speed ripple," IEEE Trans. Energy Convers., vol. 18, no. 4, pp. 508-515, 2003.

[11] S. Djurovic, D. S. Vilchis-Rodriguez, and A. C. Smith, "Supply induced interharmonic effects in wound rotor and dfigs," IEEE Trans. Energy Convers., vol. 30, no. 4, pp. 1397-1408, 2015.

[12] G. Mv and N. P. Padhy, "PHIL experimentation on fault ride through behavior of doubly fed induction generator based wind system in the presence of fault current limiter," IEEE Trans. Ind. Appl., pp. 1-1, 2020.

[13] N. Jabbour, E. Tsioumas, C. Mademlis, and E. Solomin, "A highly effective fault-ride-through strategy for a wind energy conversion system with DFIG," IEEE Trans. Power Electron., pp. 1-1, 2020.

[14] H. Nian and Y. Jiao, "Improved virtual synchronous generator control of DFIG to ride-through symmetrical voltage fault," IEEE Trans. Energy Convers., pp. 1-1, 2019.

[15] G. Pannell, D. J. Atkinson, and B. Zahawi, "Analytical study of gridfault response of wind turbine doubly fed induction generator," IEEE Trans. Energy Convers., vol. 25, no. 4, pp. 1081-1091, Dec. 2010.

[16] Y. Deng, Z. Xing, and Q. Zhang, "Analysis of electromagnetic transient characteristics of DFIG under grid voltage swell," CPSS Trans. Power Electron. Appl., vol. 3, no. 2, pp. 111-118, Jun. 2018.

[17] S. Tohidi, "Analysis and simplified modelling of brushless DFIM in synchronous mode of operation," IET Elect. Power Appl., vol. 10, no. 2, pp. 110-116, 2016.

[18] M. Yousefian, H. A. Zarchi, and H. Gorginpour, "Modified steady-state modelling of brushless DFIG taking core loss components into account," IET Elect. Power Appl., vol. 13, DOI 10.1049/iet-epa.2019.0133, no. 9, pp. 1402-1412, 2019.

[19] Z. M. Salameh and L. F. Kazda, "Analysis of the double output induction generator using direct three-phase model part ii - harmonic analysis," IEEE Trans. Energy Convers., vol. EC-2, no. 2, pp. 182-188, 1987.

[20] M. Zhou, Z. Dong, H. Li, C. Gan, G. Li, and Y. Liu, "Coordinated control of dfig based wind farms and sgs for improving transient stability," IEEE Access, vol. 6, pp. 46844-46855, 2018.

[21] D. Kim, Y. Moon, and H. Nam, "A new simplified doubly fed induction generator model for transient stability studies," IEEE Trans. Energy Convers., vol. 30, no. 3, pp. 1030-1042, Sep. 2015.

[22] F. Maurer and J. K. Nøland, "An analytical model for detailed transient fault analysis of doubly-fed induction machines," in Proc. Int. Conf. Electr. Mach. (ICEM), vol. 1, pp. 1287-1293, 2020.

[23] K. W. Kanelis, "Analytical equations of the induction machine," in Proc. Int. Conf. Elect. Mach. (ICEM), pp. 2854-2860, Sep. 2016.

[24] J. Chatelain, "Machines electriques, volume x," Traite d'electricite series, Presses Polytechniques. PPUR, Lausanne, ppur Edition, 2013.

[25] I. Canay, "Extended synchronous-machine model for the calculation of transient processes and stability," Electric machines and Electromechanics, vol. 1, no. 2, pp. 137-150, 1977.

[26] F. M. Maurer, "Electrical effects in winding of large electrical AC machines application to advanced large size DFIM," EPFL, Tech. Rep., 2019.

[27] F. Maurer, M. T. Xuan, and J. Simond, "Two full parameter identification methods for synchronous machine applying DC-decay tests for a rotor in arbitrary position," IEEE Trans. Ind. Appl., vol. 53, no. 4, pp. 35053518, Jul. 2017.

[28] F. Maurer, "Determination des parametres de la machine synchrone par essais "DC-Decay"," Master thesis, EPFL, 2009. 